\title{
A fast and accurate method to detect allelic genomic imbalances underlying mosaic rearrangements using SNP array data
}

\author{
Juan R González ${ }^{1,2,3^{*}}$, Benjamín Rodríguez-Santiago ${ }^{4,5}$, Alejandro Cáceres ${ }^{1,2}$, Roger Pique-Regi ${ }^{6}$, \\ Nathaniel Rothman ${ }^{7}$, Stephen J Chanock ${ }^{7,8}$, Lluís Armengol ${ }^{9}$ and Luis A Pérez-Jurado ${ }^{4,5}$
}

\begin{abstract}
Background: Mosaicism for copy number and copy neutral chromosomal rearrangements has been recently identified as a relatively common source of genetic variation in the normal population. However its prevalence is poorly defined since it has been only studied systematically in one large-scale study and by using non optimal adhoc SNP array data analysis tools, uncovering rather large alterations (> $1 \mathrm{Mb}$ ) and affecting a high proportion of cells. Here we propose a novel methodology, Mosaic Alteration Detection-MAD, by providing a software tool that is effective for capturing previously described alterations as wells as new variants that are smaller in size and/or affecting a low percentage of cells.

Results: The developed method identified all previously known mosaic abnormalities reported in SNP array data obtained from controls, bladder cancer and HapMap individuals. In addition MAD tool was able to detect new mosaic variants not reported before that were smaller in size and with lower percentage of cells affected. The performance of the tool was analysed by studying simulated data for different scenarios. Our method showed high sensitivity and specificity for all assessed scenarios.

Conclusions: The tool presented here has the ability to identify mosaic abnormalities with high sensitivity and specificity. Our results confirm the lack of sensitivity of former methods by identifying new mosaic variants not reported in previously utilised datasets. Our work suggests that the prevalence of mosaic alterations could be higher than initially thought. The use of appropriate SNP array data analysis methods would help in defining the human genome mosaic map.
\end{abstract}

\section{Background}

Microarray platforms based on Single Nucleotide Polymorphisms (SNP arrays) are powerful tools in the research of genomic structural variation because they allow the integration of genotype and copy-number information. Researchers can simultaneously identify both copy number and copy number neutral changes, using the $\log 2$-ratio (LRR) intensity signal and the allele genotyping of the probes [1-6]. While SNP arrays have been effectively used in the study of copy number variation (CNV) and single nucleotide polymorphism (SNP) genotyping, only recently they have been utilised to

\footnotetext{
* Correspondence: jrgonzalez@creal.cat

'Center for Research in Environmental Epidemiology (CREAL), Doctor

Aiguader 88, Barcelona 08003, Spain

Full list of author information is available at the end of the article
}

identify the mosaic occurrence of copy number and copy neutral genomic abnormalities [7]. Genetic mosaicism is recognized as the presence of two or more different cell populations with different genotypes in one single individual, developed from a single fertilized egg. Such genetic abnormalities may result from a mutation during development that is propagated to only a subset of the adult cells. Somatic mosaicism for chromosomal rearrangements has been recently described on the basis of comparative analysis of differentiated human tissues from adult individuals [8] and divergence between identical twins [9]. Moreover it is well known that some mosaic abnormalities are involved in multiple developmental and tissue-specific disorders [10-16]. Despite of all these evidences, the frequency and extent of chromosomal mosaicism in adult normal population has been

\section{Ciomed Central}


estimated only recently for the first time [17], and its real contribution to intra- and inter-individual genome variation is yet to be determined. For helping in that purpose, specialized algorithms and data analysis tools aimed at calling the mosaic occurrence of structural variation are badly needed.

Mosaic events can be captured by analysing SNP array data, specially from assessing multiple clusters of heterozygous alleles showing B allele frequency (BAF) and LRR values different from the those expected for regular heterozygous deletions, duplications or loss of heterozygosis events (See Additional File 1 - Figure S1 for examples of different types of mosaic rearrangements). Two recent studies have demonstrated that structural variants occurring in mosaicism are more frequent than expected, and thus they may play a relevant role in human diversity and disease susceptibility [7,17]. While both studies used Illumina SNP array data, only Rodríguez-Santiago et al. (2010) used tools for discovering occurrences in a systematic way. Their approach may, however, result in the underestimation of mosaic prevalence in two challenging situations: 1) small rearrangements and 2) rearrangements affecting a low percentage of cells. In addition, the used algorithm was computationally demanding ( 2 weeks to analyze about 2,000 individuals genotyped with Illumina HumanHap 1M), which constitutes a technical drawback in the analysis of high-density arrays of thousands of individuals.

Mosaicism detection can be cast as a segmentation problem [18]. In fact BAFsegmentation is a software developed for the identification of mosaicism in cancer cells based on the circular binary segmentation (CSB) algorithm [19]. The disadvantage of this procedure is the lack of a method to clearly control the false discovery rate (FDR) [20].

As an alternative segmentation method, the genome alteration detection analysis (GADA) can also identify allelic imbalances by using BAF values provided by SNP arrays [21]. This value is the fraction of the total signal due to a specific allele and it is the suited value to study allelic imbalances underlying mosaicism for genomic rearrangements. Compared to circular binary segmentation (CBS) [19], GADA has similar accuracy, and is several orders of magnitude faster. Recently, GADA segmentation was applied to CNV calling by using LRR values from SNP array data in very large data sets with high efficiency and accuracy $[6,22]$. To overcome the specific difficulties in the identification of mosaic events from SNP arrays, we have developed Mosaic Alteration Detection-MAD method which includes both statistic (including FDR control) and bioinformatic tools to specifically analyse BAF values from SNP array data. The software presented here improves SNP array data analysis allowing the capture of mosaic copy number (deletions, duplications, aneuploidies) and copy neutral changes (uniparental disomies, UPD, namely the occurrence of two copies of a particular chromosome from the same parent), as well as regions of homozygosity due to identity-by-descent. The developed method was used to analyse Illumina HumanHap 1M SNP array data obtained from control, bladder cancer and HapMap individuals. We also compared its performance (sensitivity and specificity) with BAFsegmentation [18] under several simulated challenging scenarios like small altered regions, low percentage of mosaic cells and poor array quality. Finally, our tool was applied to SNP array data previously utilised for detecting mosaic abnormalities $[17,18]$. While all published findings were successfully identified, additional mosaic events were detected by MAD and experimentally validated afterwards. Our results suggest that improved methods can accurately capture mosaic chromosomal rearrangements using SNP array data and that their prevalence is higher than initially thought.

\section{Results and Discussion}

\section{Method development}

Figure 1 contains a scheme showing the overall performance of the MAD algorithm, using CASE571 carrying a mosaic deletion in chromosome 20 from Mb 31 to 48 , as an example. The distribution of LRR and BAF values for the individual can be visualised in Figure 1a. Two clusters of BAF values (in red) are clearly visible between MB 31 to 48 that would be absent in a standard heterozygous deletion. Therefore, the abnormality could be detected by the deviation of the BAF signal from the expected values typical for non-altered homozygous ( 1 or 0 ) or non-altered heterozygous probes (0.5). The difference between observed and expected BAF is denoted as b-deviation. Altered regions can be called by detecting segments with b-deviation values different from zero using algorithms such as GADA and CBS. Segmentation methods usually assume that the data are normally distributed. However this is not the case for b-deviation as Figure $1 \mathrm{~b}$ demonstrates. The further variable $\Phi^{-1}$ (Figure 1c), which is a probit transformation on b-deviation having a normal distribution, can be applied to perform the segmentation procedure (Figure 1d) controlling the FDR parameter. Once the segments have been called by assessing BAF values as described above, the average LRR of the called segment is calculated to help in determining whether the abnormality is affecting the copy number (Figure 1e).

\section{New mosaic rearrangements from previously utilised SNP array datasets HapMap individuals}

To check how the algorithm works with real data MAD tool was applied to data obtained from lymphoblastic 


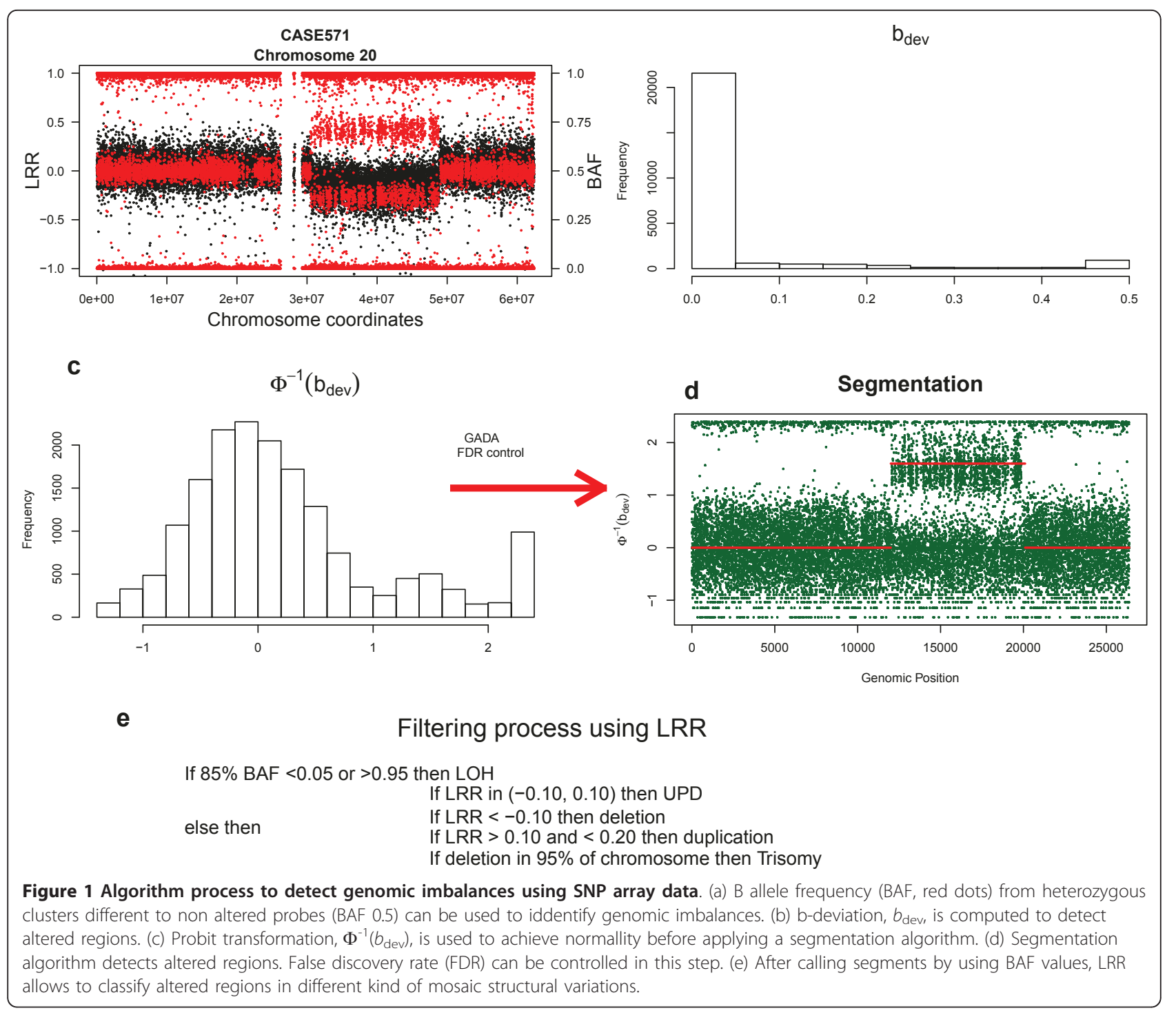

cell line DNA of 125 individuals from HapMap population (60 CEU, 60 YRI and 5 replicates) hybridized with Illumina HumanHap 1M SNP array, available at public repositories http://www.hapmap.org. For MAD, parameters were set to $T=8, a=0.8$ (see Method section for a definition of these two parameters and Additional File 2 for a table including the recommended settings for being used in a preliminary scan) and the minimum probe length for detection at MinSegLen $=300$, meaning the algorithm is able to detect altered regions $>0.9 \mathrm{Mb}$ for $1 \mathrm{Mb}$ arrays. Furthermore, with $a=0.8$ finding few number of segments for each individual is expected. With these parameters, the FDR was $<0.0001$. The tool identified 9 mosaic rearrangements in 8 individuals (Table 1). Mosaic uniparental disomy was observed for chromosome 2 in one individual (NA18855). Mosaic duplications were detected in three individuals affecting chromosome 2 (NA10857), chromosome 19 (NA11882) and chromosome 8 (NA18972). Gains of the entire chromosome compatible with mosaic trisomies were observed in 4 individuals (NA11236, NA12248, NA12875 and NA19193) involving chromosomes 15, 9 and 14, 2 and 12, respectively and different percentage of mosaic cells (see Additional File 3). In addition the performance of MAD was compared to BAFsegmentation using default parameters in the HapMap individuals analysed. Compared to MAD, BAFsegmentation algorithm was unable to detect 2 alterations, trisomies with very low proportion of affected cells (see Figure 2 for one case). The obtained results are consistent with the simulation analysis performed in the present work where BAFsegmentation showed less sensitivity for low mosaic cell proportions (see section below). 
Table 1 Summary of mosaic structural variants identified in HapMap individuals by using MAD tool.

\begin{tabular}{lcccccc}
\hline Sample & BAFsegmentation? & Chr & Event & Start & End & Size (Mb) \\
\hline NA10857 & Yes & 2 & Duplication & $59,505,990$ & $60,622,482$ & 1.1 \\
NA11882 & Yes & 19 & Duplication & $57,313,456$ & $63,802,440$ & 6.7 \\
NA12236 & Yes & 15 & Trisomy & pter & qter \\
NA12248 & No & 14 & Trisomy & pter & qter \\
NA12248 & No & 9 & Trisomy & pter & qter \\
NA12875 & Yes & 2 & Trisomy & pter & qter & $94,755,295$ \\
NA18855 & Yes & 2 & UPD & pter & 9,260 \\
NA18972 & Yes & 8 & Duplication & $4,669,277$ & $5,914,260$ & qter \\
NA19193 & Yes & 12 & Trisomy & pter & & 1.2 \\
\hline
\end{tabular}

The second column indicates whether BAFsegmentation algorithms also found the alteration.

\section{Control and bladder cancer individuals}

The tool was also used to re-analyse previously defined and validated mosaic rearrangements reported elsewhere [17]. A selected subset of 31 mosaic rearrangements among 58 samples was initially studied. Parameters were set to $T=8, a=0.8$ and MinSegLen $=1500$ that are predicted to have a FDR of 0.0001 .
Using these settings the tool was able to detect all previously defined mosaic rearrangements but one (30/31). Remarkably, the algorithm did not provide any falsepositive calls. The false-negative result was a small deletion on chromosome 20 only comprised by 248 probes (that is not possible to be detected with MinsegLen = $1500)$. Nonetheless, this alteration was detected by

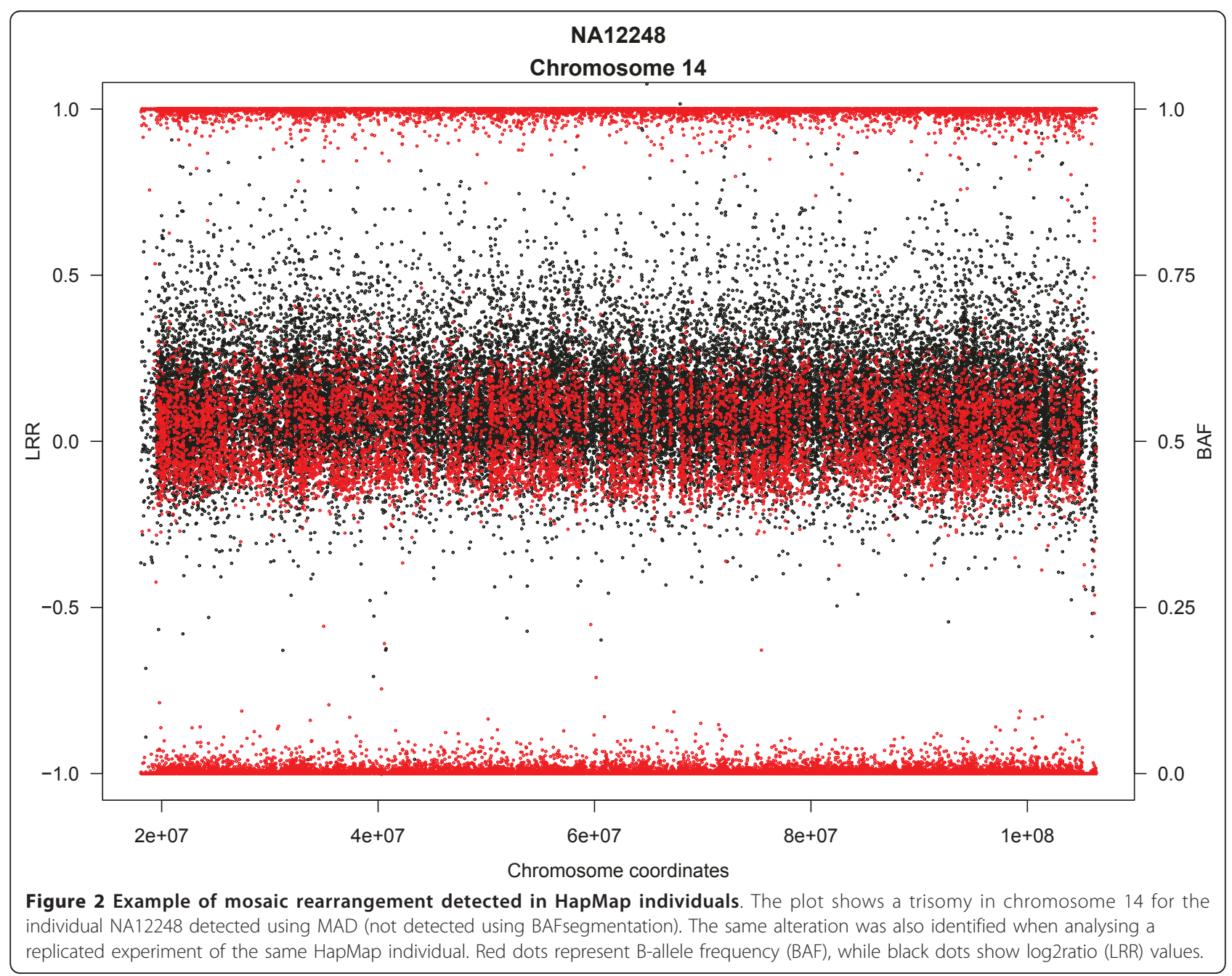


changing MinSegLen to 200, and no other abnormality was found in this sample by using this setting. Overall, the predicted FDR using MinSegLen $=200$ is 0.001 . The MinSegLen is a parameter that can be adjusted depending on probe coverage of the array, and it can be reduced for hihg-density arrays such as Illumina HumanHap 1M. The complete dataset of 1991 samples was studied afterwards [17]. We set MinSegLen $=100$ and $T=8$, to increase power detection at the expense of higher FDR (0.05). The algorithm detected 7 new segments not identified either with previous tools or with BAFsegmentation (Table 1 and Additional File 1 - Figures S5 to S11). These abnormalities were smaller in size than those previously reported, with the minimum size of $\sim 500 \mathrm{~Kb}$ (See Additional File 1 - Figure S5 for an example) which represents an improvement in the detection of smaller events. We experimentally validated the mosaic abnormalities in all 6 available DNA samples by multiple ligase-dependent probe amplification (MLPA) (Table 2 and Additional File 1 - Figures S12 to S17). The algorithm also detected 98 duplication segments ranging in size from $\sim 0.2$ to $~$ $4 \mathrm{Mb}$ with average LRR $<0.15$ suggestive of possibly being mosaics (Additional File 4).

\section{Simulation Studies}

Several simulation studies were run to further assess the performance of MAD and BAFsegmentation analysis tools. Specifically, eight scenarios were considered depending on i) the percentage of affected cells with the altered region: $10 \%$ and $20 \%$; ii) the length of the alteration: small and large; and iii) the quality of data: good and noisy. The BAF value was simulated for 20,000 SNPs using a log-normal distribution with mean 0 for AA homozygous and mean 1 for BB homozygous probes. A normal distribution with mean 0.5 was used to generate heterozygous markers. Different quality data was generated varying the variance of these distributions ( 0.03 for good quality and 0.1 for noisy). The percentage of cells with an abnormal region was simulated by changing the mean value for heterozygous probes $(0.55$ and 0.60 for $10 \%$ and $20 \%$ of affected cells, respectively). Finally, abnormal large regions were generated by modifying 10,000 markers, while small aberrant regions contained 1,000 probes. These simulations allowed the calculation of: 1) the ROC curve (True-positive Vs False positive rates); 2) the FDR; and 3) differences in sensitivities of MAD and BAFsegmentation. The results were based on 1,000 simulations.

\section{True-positive (TPR) and False-positive (FPR) rates}

The performance of MAD algorithm was examined under changes in the parameters, $a \in\{0.2,0.8\}$ and $T \in$ $\{2.5,3,3.5,4,4.5,5\}$, that control its sensitivity and specificity. Figure 3 shows the ROC curve (e.g. TPR vs FPR) for different simulated scenarios and for the case of having moderate to large altered regions. As expected, the TPR improves when both quality data and percentage of affected cells increase. The performance was almost perfect (e.g. area under the ROC curve is near 1) when quality data was good and the degree of mosaicism was at least 20\% (continuous red line). Similar results were obtained when analyzing simulated segments with less density (1,000 abnormal SNPs Additional File 1 - Figure S4).

\section{False discovery rate (FDR)}

As a significant feature MAD incorporates a general method to control FDR. This method was validated by using simulated datasets and comparing estimated with expected FDR values. Figure 4 shows the FDR comparison for different scenarios under $a=0.8$. A good agreement between simulated and estimated FDR in all situations was observed: the FDR decreased when either $T$, the percentage of affected cells or data quality increased. Similar results were obtained for $a=0.2$, where the FDR is larger than the previous case, as expected.

\section{Comparison with BAFsegmentation}

The performance of MAD and BAFsegmentation tools was studied by simulating low cell proportion of mosaic alterations. A single segment of 1000 probes within a

Table 2 Eight new mosaic events identified in SNP array data from individuals previously analysed in RodriguezSantiago et al. (2010).

\begin{tabular}{|c|c|c|c|c|c|c|}
\hline Sample & Chr & Event & Start & End & Size (Mb) & MLPA validation ${ }^{1}$ \\
\hline CONTROL870 & 2 & Deletion & $25,056,172$ & $25,570,101$ & 513,929 & Yes \\
\hline CONTROL1210 & 2 & Deletion & $25,312,050$ & $26,013,444$ & 701,394 & Yes \\
\hline CASE623 & 4 & Duplication & $158,590,621$ & $159,723,224$ & $1,132,603$ & Yes \\
\hline CASE741 & 6 & Duplication & $162,534,963$ & $163,052,519$ & 517,556 & NA \\
\hline CASE526 & 7 & Deletion & $138,459,817$ & $139,610,819$ & $1,151,002$ & Yes \\
\hline CASE508 & 11 & Duplication & $122,663,431$ & $123,143,910$ & 488,481 & Yes \\
\hline CONTROL413 & 15 & Duplication & $48,349,855$ & $48,838,336$ & 517,556 & Yes \\
\hline
\end{tabular}

The new abnormalities observed were smaller than those previously reported. Figures are available in the Additional File 6 - Figures S5 to S11.

${ }^{1}$ Additional File 5 (Section 4) shows the MLPA electropherograms and control comparisons. 


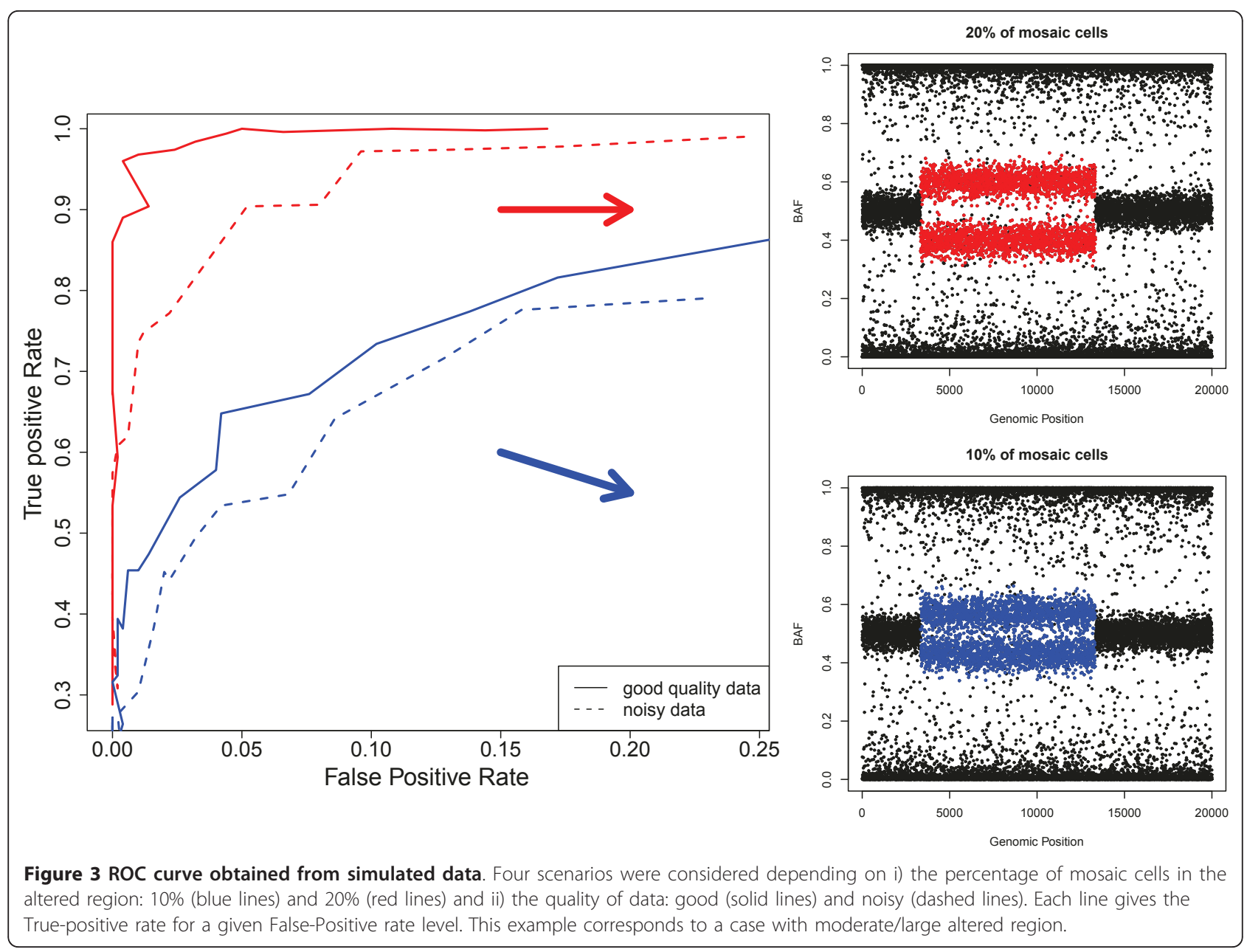

DNA chunk of $20-\mathrm{Kb}$ in length was simulated 200 times for different proportion of mosaic cells $(0,0.01, \ldots 0.15)$. Parameters of both algorithms were fixed such as optimum specificity was achieved for both methods across the whole range. In other words, the algorithms were adjusted for not finding any segment which was completely disjointed from the one simulated. In particular, this was satisfied with $a=0.5, T=2$ and MinSegLen = 900 for MAD, and default values for BAFsegmentation. The comparison was assessed using the sensitivity of each method by measuring the proportion of identified segments covering at least $50 \%$ of the simulated segment.

Overall MAD showed a better performance when compared to BAFsegmentation as can be seen in Figure 5. BAFsegmentation achieved good sensitivity in the range of mosaic cell proportions $>0.07$, and null sensitivity for values $<0.05$. On the other hand, despite the lower sensitivity of MAD in the range $(0.07,0.15)$, there is an important amount on sensitivity captured in low values $(0.02,0.05)$ and a high sensitivity $(0.98)$ at 0.15 .
The overall performance of both methods can be compared from the areas under each curve. In the case of the MAD curve the estimation of this area, normalized by the area of the perfect sensitivity curve $(y=1)$, is $0.109 / 0.15=0.73$; whereas for BAFsegmentation this area is smaller (0.63). Therefore, under this scenario, MAD showed better sensitivity over the whole range of mosaic cell proportions. In addition, the computational time for analyzing the 58 samples described in previous sections was $3 \mathrm{~min} 15 \mathrm{sec}$ when using MAD, while BAFsegmentation needed $42 \mathrm{~min} 50 \mathrm{sec}$.

\section{Conclusions}

The accurate and appropriate analysis of SNP array data of genomic DNA from multiple cells allows for the identification of genomic changes occurring in mosaicism and subsequently for the estimation of the affected cell proportion. The assessment of this increasingly recognised type of genetic variation is relevant to define its impact over human diversity and clinical phenotypes. In this study, we have implemented the so called MAD 


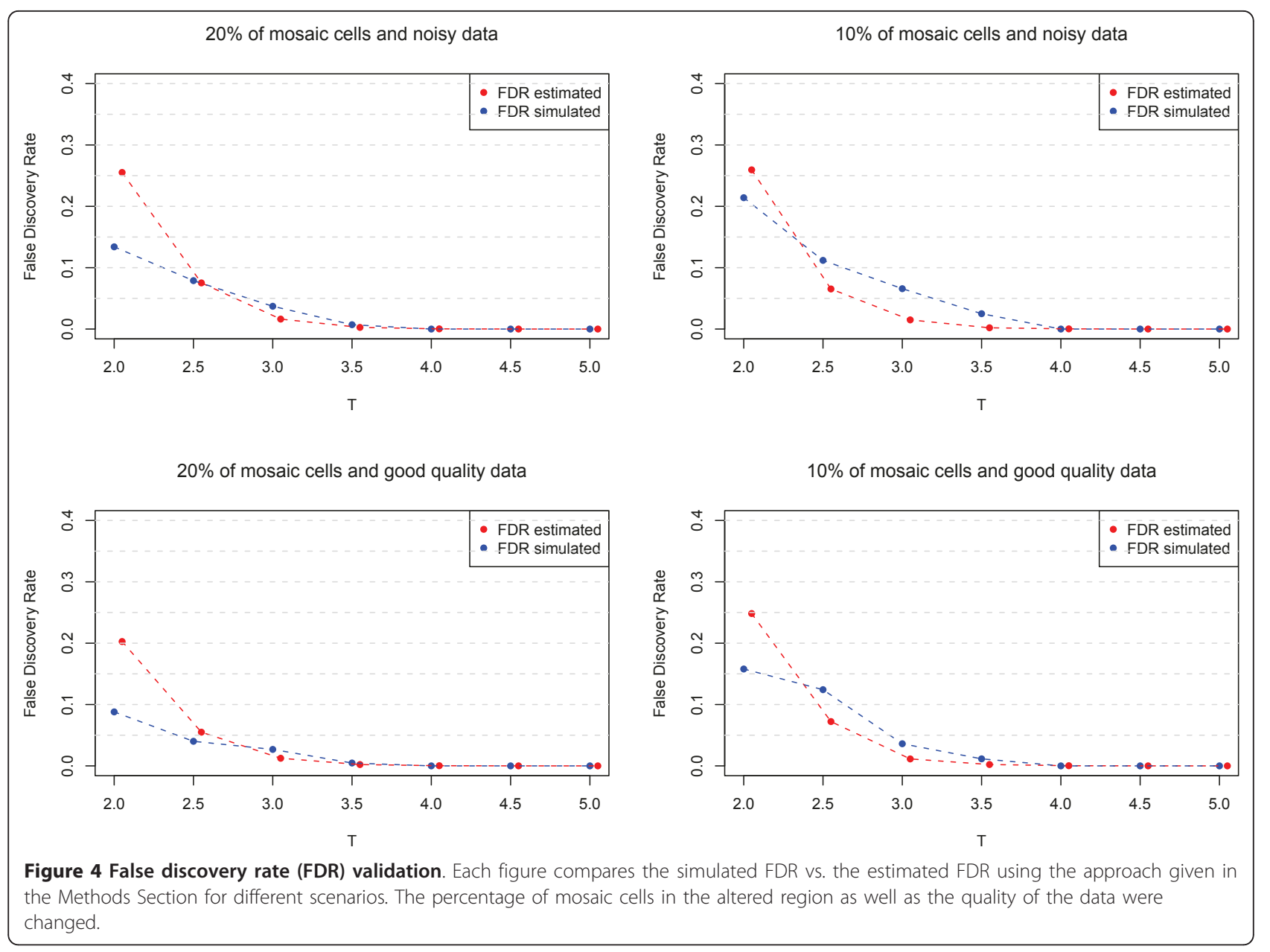

tool to detect mosaic events from SNP arrays using the BAF value as a powerful parameter to detect the allelic imbalances that underlie mosaic alterations. Our method was successful in finding previously defined mosaic chromosomal alterations, and able to detect additional events in the same data set $[17,18]$, which suggests a higher sensitivity for MAD. Remarkably, the tool was able to find mosaic rearrangements of smaller size $(\sim$ $500 \mathrm{~Kb})$ and events affecting a lower proportion of cells, uncalled when using other algorithms.

The easy manipulation of the parameters $a$ and $T$ offers flexibility to the optimization of MAD for a wide range of circumstances. In contrast, a greater optimization of BAFsegmentation has yet to be developed for truly assessing the performace of the method, especially for small and low proportion of cells affected Another significant advantage of MAD is that the FDR can be directly handled by performing setting changes in its parameters. High sensitivity and low FDR are essential in assessing the prevalence of mosaic events in the available datasets. We have shown how to estimate the FDR from the segmentation output and, in our simulation studies, how close is such estimate is very close to the expected one. However, it is important to note that FDR also depends on the window for minimum probe length (MinSegLen). One possible limitation of our method is the increase in the number of false positives when reducing the (MinSegLen) for analysing arrays with lower probe coverage.

An unexpectedly increased number of duplications with respect to deletions were called by MAD. Most of these calls were not treated as mosaic because their features (size, LRR, Bdev, ...) or their plots did not suggest mosaic occurrence (see Additional File 4). While the distinction between constitutional and mosaic events is quite evident for deletions and UPDs, due to the finding of complete loss of heterozygosity only in non-mosaic rearrangements, in the case of copy number gains (duplications, trisomies) it is not so straightforward because the presence of heterozygous probes is expected. Due to the technical limitations in array platforms the change in copy number from 2 to 3 or an intermediate number could be in the same sensitivity range thus providing LRR and BAF array values similar 


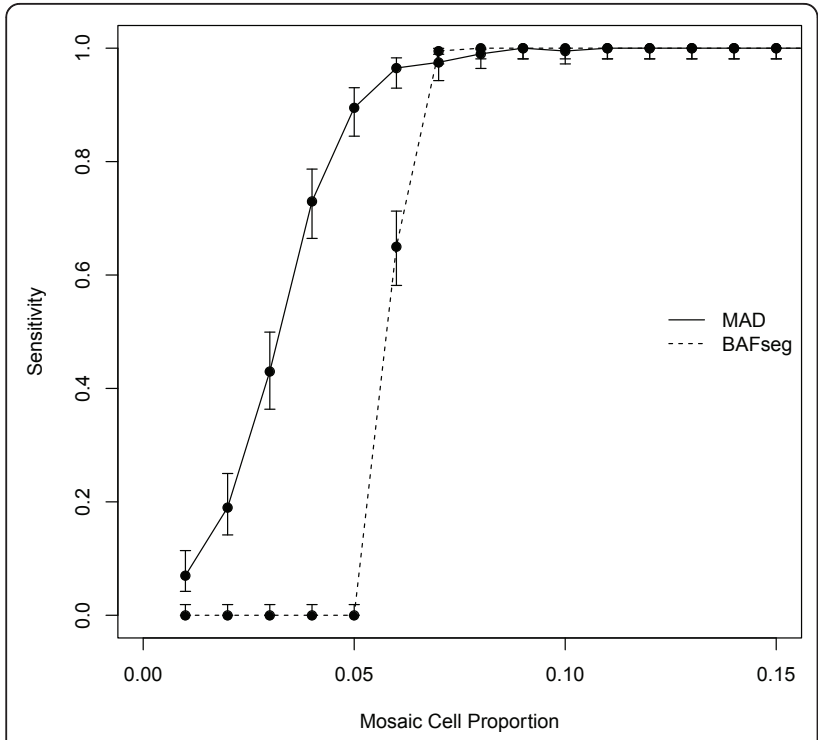

Figure $\mathbf{5}$ Sensitivity as a function of mosaic cell proportion. Low proportion of cells affected with the abnormality reduces the sensitivity to identify a $1 \mathrm{kB}$ mosaic alteration, in a $20 \mathrm{kB}$ region of 200 simulated individuals. Overall MAD showed a better performance when compared to BAFsegmentation.

for both normal gain dosage changes (2 to 3 i.e.) and mosaic changes occurring in a subset of cells. A comparison between MAD analysis and CNV calling process using PennCNV and filtering procedures revealed that 30 MAD duplications were absent in CNV dataset when a statistic filter was applied [23,24]. The 98 duplications showing any overlap in both datasets were $59 \%$ identical on average (range: $3 \%$ - 100\%) (see Additional File 4). These results may suggest on one hand that MAD software seems to be more effective in calling duplications than typical CNV calling procedures and, in the other hand, that some duplication calls observed using MAD may be non mosaic alterations.

Reanalysis of the previously assessed SNP array data in [17] demonstrates that there are hidden genomic mosaic events that cannot be detected by using ad-hoc routines. The fact that such genomic allelic imbalances can be smaller and present in a lower proportion of cells, emphasizes the need of improving and using more powerful analytical methods. However, an open question regarding the detection limit remains partially dependent on the array resolution given that no gold standard exists to define the false negative rate for most rearrangements (other than FISH for large segmental or complete aneuplodies). While we have shown that MAD can successfully detect small and very low mosaicism degree events and that the prevalence of mosaicism can still be higher, further improvements are needed in the analysis and experimental techniques. Mosaicism is an unexpected source of genetic variation that is still underexplored.
The study of existing and future SNP array datasets, as well as the application of similar algorithms for allelic imbalance detection to next-generation sequencing data, will provide new clues about the impact of such genetic variation over phenotypic differences in the common population as well as its influence on disease.

\section{Methods \\ Analysis methods \\ Data}

The simulated dataset was generated by varying i) the percentage of cells affected by a deletion type CNV (varying shift in B allele frequency to be $10 \%$ or $20 \%$ ); ii) the length of the rearrangement, from large (half chromosome) to small (5\% of the chromosome); and iii) the quality of the hybridization BAF signal data, that were defined as good (Additional File 1 - Figure S2) or noisy experiments (Additional File 1 - Figure S3).

The Illumina data set from HapMap individuals comprised 120 individuals and 5 replicates and were retrieved from http://www.illumina.com/. The data includes $60 \mathrm{CEPH}$ (CEU) and 60 Yoruban (YRI) unrelated samples. The Illumina $1 \mathrm{M}$ HumanHap SNP array integrates about 1.0 million probes. Data were normalized by using Illumina BeadStudio software.

A subset of Illumina 1M HumanHap SNP array data obtanined from 58 individuals who participated in the Spanish Bladder Cancer study [25] kindly provided by the NCI Core Genotyping Facility was also analysed. These data have been already analyzed for mosaic events using different ad-hoc tools and manual curation [17]. All reported mosaic rearrangements were validated by additional molecular techniques such as multiplex ligation probe-dependent amplification (MLPA) and microsatellite marker analysis and/or FISH [17].

\section{Algorithm}

Allelic genomic imbalances (different proportions of the two homologous chromosome regions) can be detected from the BAF signal data (Figure 1). The transformed bdeviation value was calculated for each probe to detect genomic imbalances. The b-deviation of a probe, $b_{\mathrm{dev}}$, is defined as the deviation from the expected BAF given the genotype,

$$
b_{\mathrm{dev}}=\left\{\begin{array}{l}
\min (\mathrm{BAF}, 1-\mathrm{BAF}) \text { for homozygotes } \\
\mathrm{abs}(\mathrm{BAF}-0.5) \text { for heterozygotes }
\end{array}\right.
$$

Genomic imbalanced regions are captured by selecting SNPs with $b_{\text {dev }}$ different from 0 . This is a segmentation problem that can be tackled with methods already available, most of which assume normality in the signal. Thus, the probit transformation, $\Phi^{-1}$, was used

$$
\Phi^{-1}\left(b_{\mathrm{dev}}\right)= \begin{cases}0, & b_{\mathrm{dev}}=0 \\ \sqrt{2} \operatorname{erf}^{-1}\left(2 b_{\mathrm{dev}}-1\right), & b_{\mathrm{dev}} \in(0,1)\end{cases}
$$


where erf denotes the error function (Figure 1C). Segmentation is then performed on regions with $\Phi^{-1} \neq$ 0 using GADA which detects altered segments in two steps. The first step is a Sparse Bayesian Learning process (SBL) that generates a list of candidate breakpoints and segment means while trying to strike an optimal balance between model fit and model sparseness (the number of breakpoints). The SBL step is driven by two prior parameters $a$ and $b$ and they are directly controlled by the user. Typically $b$ is set to zero as an uninformative prior, so sparseness is solely controlled by $a$, taking values between 0.2 and 0.8 . By increasing $a$ the algorithm has higher sensitivity but also higher FDR, which can be further adjusted in the second step. The significance of each called breakpoint segment is assessed with a $t$-statistic computed with the parameter estimatation provided by the SBL step. This statistic is a function of the segment mean and variance. The second step is then a backward elimination (BE) process which removes breakpoints with a level of significance (t statistic) lower than the userdefined threshold, [21] showed that, under the null hypothesis, if a segment is copy normal, the t-statistic distribution is normal across the segments. The ranking of breakpoints with the adjustment of $\mathrm{T}$ is obtained with very low computational cost and can be used to control the FDR.

After calling segments by assessing BAF values the average LRR is used to carry out a preliminary classification of the called segment into different types of mosaic rearrangements: UPD was considered for LRR $\in\{-0.10$, $0.10\}$, a deletion when LRR $<-.10$, a duplication for LRR $>0.10$ and a Trisomy if a deletion was observed in more than $95 \%$ of the chromosome. These values should not be absolute but relative to the average LRR of the entire diploid genome (exluding $\mathrm{X}$ and $\mathrm{Y}$ chromosomes) and the rest of the chromosome data.

\section{False Discovery Rate estimation}

Recommendations on how to choose $T$ and $a$ parameters to control both sensitivity and FDR, based on simulations and specific data (i.e., Affymetrix $500 \mathrm{~K}$, Illumina 550) have been given elsewhere [21]. Here, we propose a general method to control the FDR independent of reference arrays and resolution, derived from a previously reported one [26].

The hypothesis of no alteration for the $i$-th segment, $S_{i}$, can be stated as

$$
H_{0}^{i}: \mu_{i}=0,
$$

where $\mu_{i}$ denotes the mean of $\Phi^{-1}\left(b_{d e v}\right)$ across all $n_{i}$ probes in $S_{i}$. For each $S_{i}$, GADA provides a statistic, $t_{i}$. Under the null hyphotesis the distribution of $t_{i}$ is $N(0$, 1) for a large number of probes, whereas for a small number of probes it follows a $t$-Student distribution, $t_{v}$, with $v=n_{i}-1$ degrees of freedom. If $p_{k}=P\left(t>\left|t_{i}\right|\right)$ and $t_{i}$ are independent from each other, for a given $T$, a conservative estimator of the genome-wide FDR is

$$
\operatorname{FDR}(T)=\frac{N P\left(t_{v}>T\right)}{\sum_{k} n_{k} I_{p k} \leq P\left(t_{v}>T\right)},
$$

where $N$ is the total number of probes in the array.

\section{Software}

MAD is included in the R-GADA software [27] that is available at

http://www.creal.cat/jrgonzalez/software.htm. A detailed tutorial describing how to analyze SNP array data is available in the Additional File 5.

\section{Experimental validation}

DNA samples were obtained from 6 out of 7 individuals showing mosaic abnormalities in Table 2. Validation of algorithm findings was carried out by using multiplex ligation-dependent probe amplification (MLPA) technique [28]. The MLPA reactions were carried out essentially as described previously [28] with slight modifications [29]. We used both visual examination of the electropherograms and the relative peak height (RPH) method recommended by MRC-Holland for data analysis [30]. The complete list and details of used MLPA probes is shown in Additional File 6.

\section{Additional material}

Additional file 1: File including figures for examples of simulated data sets, some simulation results and new mosaic abnormalities detected using MAD in SNP arrary data previously analyzed with ad-hoc tools (Rodriguez-Santiago et al., 2010).

Additional file 2: Recommended parameters for a preliminary scan using different Illumina platforms

Additional file 3: Comparison between MAD findings and chromosomal abnormalities previously described in HapMap individuals also analysed in Redon et al., Nature, 2006

Additional file 4: Additional analysis using PennCNV to discard mosaic detected with MAD with consitutional duplications. Additional file 5: User's guide of an R package that implements $M A D$ algorithm including some real data examples

Additional file 6: List and details of used MLPA probes for validating new mosaic rearrangements.

\section{Acknowledgements}

We thank Dr. Francisco X. Real (CNIO, Spain), Dr. Nuria Malats (CNIO, Spain), Dr. Manolis Kogevinas (CREAL, Spain) and Dr. Debra T. Silverman (NCI, U.S.A.) for contributing data and information from the Spanish Bladder Cancer study. This work has been supported by the Spanish Ministry of Science and Innovation (MTM2008-02457 to JRG), the Fondo de Investigación Sanitaria (grant PI076832 to LAP-J), the intramural research program of the $\mathrm{NIH}, \mathrm{NCl}$ (to SJC and NR) and the Asociación Española Contra el Cáncer (AECC) (to FXR and LAP-J). B. Rodríguez-Santiago was supported by a postdoctoral fellowship (FIS CD06/00019) of the Fondo Investigación Sanitaria, Spain. 


\section{Author details}

${ }^{1}$ Center for Research in Environmental Epidemiology (CREAL), Doctor Aiguader 88, Barcelona 08003, Spain. ${ }^{2}$ Institut Municipal d'Investigació Mèdica (IMIM), Doctor Aiguader 88, Barcelona 08003, Spain. ${ }^{3} \mathrm{CIBER}$ Epidemiología y Salud Pública (CIBERESP), Spain. ${ }^{4}$ Dept. de Ciències Experimentals i de la Salut, UPF, Barcelona 08003, Spain. ${ }^{5}$ CIBER de Enfermedades Raras, CIBERER, Spain. ${ }^{6}$ Department of Human Genetics, University of Chicago, IL 60637, USA. ${ }^{7}$ Division of Cancer Epidemiology and Genetics, National Cancer Institute, Bethesda, MD 20852-4907, USA. ${ }^{8}$ Core Genotyping Facility, SAIC-Frederick, Frederick, MD 21702, USA. ${ }^{9}$ Quantitative Genomic Medicine Laboratories, Ltd (qGenomics), Doctor Aiguader 88, Barcelona 08003, Spain.

\section{Authors' contributions}

$J R G, B R-S, L A$ and $L A P-J$ conceived the idea of using $B$ allele frequency value and a segmentation algorithm to detect mosaic alterarions from SNP array data. JRG and BR-S analyzed real data, and co-wrote the manuscript draft. BR-S and LAP-J interpreted and further analyzed real data results. JRG and RP-R implemented MAD algorithm and FDR procedure. JRG and AC designed, performed and interpreted simulation studies. NR, SJC and LAP-J supervised the project and participated in producing part of the array data analysed in the manuscript. All authors read and approved the final manuscript.

\section{Competing interests}

The authors declare no conflict of interest, excepting LA and LAPJ, executive director and member of the scientific advisory board of the qGenomics company, respectively.

Received: 6 December 2010 Accepted: 17 May 2011

Published: 17 May 2011

\section{References}

1. Sebat J, Lakshmi B, Troge J, Alexander J, Young J, Lundin P, Maner S, Massa H, Walker M, Chi M, Navin N, Lucito R, Healy J, Hicks J, Ye K, Reiner A, Gilliam TC, Trask B, Patterson N, Zetterberg A, Wigler M: Large-scale copy number polymorphism in the human genome. Science 2004, 305(5683):525-8.

2. lafrate AJ, Feuk L, Rivera MN, Listewnik ML, Donahoe PK, Qi Y, Scherer SW, Lee C: Detection of large-scale variation in the human genome. Nat Genet 2004, 36(9):949-51

3. Tuzun E, Sharp AJ, Bailey JA, Kaul R, Morrison VA, Pertz LM, Haugen E, Hayden $\mathrm{H}$, Albertson D, Pinkel D, Olson MV, Eichler EE: Fine-scale structural variation of the human genome. Nat Genet 2005, 37(7):727-32.

4. Redon R, Ishikawa S, Fitch KR, Feuk L, Perry GH, Andrews TD, Fiegler H, Shapero MH, Carson AR, Chen W, Cho EK, Dallaire S, Freeman JL, Gonzalez JR, Gratacos M, Huang J, Kalaitzopoulos D, Komura D, MacDonald JR, Marshall CR, Mei R, Montgomery L, Nishimura K, Okamura K, Shen F, Somerville MJ, Tchinda J, Valsesia A, Woodwark C, Yang F, Zhang J, Zerjal T, Armengol L, Conrad DF, Estivill X, Tyler-Smith C, Carter NP, Aburatani $\mathrm{H}$, Lee $\mathrm{C}$, Jones KW, Scherer SW, Hurles ME: Global variation in copy number in the human genome. Nature 2006, 444(7118):444-54

5. Wong KK, deLeeuw RJ, Dosanjh NS, Kimm LR, Cheng Z, Horsman DE, MacAulay C, Ng RT, Brown CJ, Eichler EE, Lam WL: A comprehensive analysis of common copy-number variations in the human genome. Am $J$ Hum Genet 2007, 80:91-104.

6. Conrad D, Pinto D, Redon R, Feuk L, Gokcumen O, Zhang Y, Aerts J, Andrews T, Barnes C, Campbell P, Fitzgerald T, Hu M, Ihm C, Kristiansson K, Macarthur D, Macdonald J, Onyiah I, Pang A, Robson S, Stirrups K, Valsesia A, Walter K, Wei J, Tyler-Smith C, Carter N, Lee C, Scherer S, Hurles M: Origins and functional impact of copy number variation in the human genome. Nature 2010, 464:704-12

7. Conlin LK, Thiel BD, Bonnemann CG, Medne L, Ernst LM, Zackai EH, Deardorff MA, Krantz ID, Hakonarson H, Spinner NB: Mechanisms of mosaicism, chimerism and uniparental disomy identified by single nucleotide polymorphism array analysis. Hum Mol Genet 2010, ddq003.

8. Bruder C, Piotrowski A, Gijsbers A, Andersson R, Erickson S, Diaz de Ståhl T, Menzel U, Sandgren J, von Tell D, Poplawski A, et al: Phenotypically concordant and discordant monozygotic twins display different DNA copy-number-variation profiles. The American Journal of Human Genetics 2008, 82(3):763-771.
9. Piotrowski A, Bruder C, Andersson R, de Ståhl T, Menzel U, Sandgren J, Poplawski A, von Tell D, Crasto C, Bogdan A, et al: Somatic mosaicism for copy number variation in differentiated human tissues. Human mutation 2008, 29(9):1118-1124.

10. Youssoufian H, Pyeritz R: Mechanisms and consequences of somatic mosaicism in humans. Nature Reviews Genetics 2002, 3(10):748-758.

11. Ballif B, Rorem E, Sundin K, Lincicum M, Gaskin S, Coppinger J, Kashork C, Shaffer L, Bejjani B: Detection of low-level mosaicism by array CGH in routine diagnostic specimens. American Journal of Medical Genetics Part A 2006, 140(24):2757-2767.

12. Cheung S, Shaw C, Scott D, Patel A, Sahoo T, Bacino C, Pursley A, Li J, Erickson R, Gropman A, et al: Microarray-based CGH detects chromosomal mosaicism not revealed by conventional cytogenetics. American Journal of Medical Genetics Part A 2007, 143(15):1679-1686.

13. Conlin L, Thiel B, Bonnemann C, Medne L, Ernst L, Zackai E, Deardorff M, Krantz I, Hakonarson H, Spinner N: Mechanisms of mosaicism, chimerism and uniparental disomy identified by single nucleotide polymorphism array analysis. Human molecular genetics 2010, 19:1263-75.

14. Lu X, Phung M, Shaw C, Pham K, Neil S, Patel A, Sahoo T, Bacino C, Stankiewicz P, Kang $S$, et al: Genomic imbalances in neonates with birth defects: high detection rates by using chromosomal microarray analysis. Pediatrics 2008, 122(6):1310.

15. Menten B, Maas N, Thienpont B, Buysse K, Vandesompele J, Melotte C, De Ravel T, Van Vooren S, Balikova I, Backx L, et al: Emerging patterns of cryptic chromosomal imbalance in patients with idiopathic mental retardation and multiple congenital anomalies: a new series of 140 patients and review of published reports. Journal of medical genetics 2006, 43(8):625.

16. Scott S, Cohen N, Brandt T, Toruner G, Desnick R, Edelmann L: Detection of low-level mosaicism and placental mosaicism by oligonucleotide array comparative genomic hybridization. Genetics in Medicine 2010, 12(2):85.

17. Rodriguez-Santiago B, Malats N, Rothman N, Armengol L, Garcia-Closas M, Kogevinas M, Villa O, Hutchinson A, Earl J, Marenne G, Jacobs K, Rico D, Tard'on A, Carrato A, Thomas G, Valencia A, Silverman D, Real F, Chanock S, Perez-Jurado L: Genome-wide Detection of Mosaic Aneuploidies and Uniparental Disomies. The American Journal of Human Genetics 2010, 87:129-138.

18. Staaf J, Lindgren D, Vallon-Christersson J, Isaksson A, Goransson $\mathrm{H}$ Juliusson G, Rosenquist R, Hoglund M, Borg AA, Ringnér M: Segmentationbased detection of allelic imbalance and loss-of-heterozygosity in cancer cells using whole genome SNP arrays. Genome Biology 2008, 9(9):R136.

19. Venkatraman $E$, Olshen $A: A$ faster circular binary segmentation algorithm for the analysis of array CGH data. Bioinformatics 2007, 23(6):657.

20. Benjamini $Y$, Hochberg $Y$ : Controlling the false discovery rate: A practical and powerful approach to multiple testing. J Roy Statist Soc Ser B 1995, 57:289-300.

21. Pique-Regi R, Monso-Varona J, Ortega A, Seeger RC, Triche TJ, Asgharzadeh S: Sparse representation and Bayesian detection of genome copy number alterations from microarray data. Bioinformatics 2008, 24(3):309-18.

22. Winchester L, Yau C, Ragoussis J: Comparing CNV detection methods for SNP arrays. Brief Funct Genomic Proteomic 2009, 8(5):353-366.

23. Wang K, Li M, Hadley D, Liu R, Glessner J, A GSF, Hakonarson H, Bucan M: PennCNV: an integrated hidden Markov model designed for highresolution copy number variation detection in whole-genome SNP genotyping data. Genome Research 2007, 17:1665-1674.

24. Itsara A, Cooper G, Baker C, Girirajan S, Li J, Absher D, Krauss RM, Myers RM, Ridker PM, Chasman DI, Mefford H, Ying P, Nickerson DA, Eichler EE: Population analysis of large copy number variants and hotspots of human genetic disease. The American Journal of Human Genetics 2009, 84:148-161.

25. García-Closas M, Malats N, Silverman D, Dosemeci M, Kogevinas M, Hein D, Tardon A, Serra C, Carrato A, García-Closas R, Lloreta J, Castaño-Vinyals G, Yeager M, Welch R, Chanock S, Chatterjee N, Wacholder S, Samanic C, Tora M, Fernandez F, Real F, N R: NAT2 slow acetylation, GSTM1 null genotype, and risk of bladder cancer: results from the Spanish Bladder Cancer Study and meta-analyses. Lancet 2005, 36:649-659.

26. Tibshirani R, Wang P: Spatial smoothing and hot spot detection for CGH data using the fused lasso. Biostat 2008, 9:18-29. 
27. Pique-Regi R, Caceres A, Gonzalez JR: R-Gada: a fast and flexible pipeline for copy number analysis in association studies. BMC Bioinformatics 2010, 11:380.

28. Schouten J, McElgunn C, Waaijer R, Zwijnenburg D, F D, Pals G: Relative quantification of 40 nucleic acid sequences by multiplex ligation -dependent probe amplification. Nucleic Acids Res 2002, 30:e57.

29. Rodríguez-Santiago B, Brunet A, Sobrino B, Serra-Juhé C, Flores R, Armengol L, Vilella E, Gabau E, Guitart M, Guillamat R, Martorell L, Valero J, Gutiérrez-Zotes A, Labad A, Carracedo A, Estivill X, LA PJ: Association of common copy number variants at the glutathione $\mathrm{S}$-transferase genes and rare novel genomic changes with schizophrenia. Mol Psychiatry 2010, 15:1023-33.

30. Slater HR, Bruno DL, Ren H, Pertile M, Schouten JP, Choo KHA: Rapid, high throughput prenatal detection of aneuploidy using a novel quantitative method (MLPA). J Med Genet 2003, 40:907-912.

doi:10.1186/1471-2105-12-166

Cite this article as: González et al: A fast and accurate method to detect allelic genomic imbalances underlying mosaic rearrangements using SNP array data. BMC Bioinformatics 2011 12:166.

\section{Submit your next manuscript to BioMed Central} and take full advantage of:

- Convenient online submission

- Thorough peer review

- No space constraints or color figure charges

- Immediate publication on acceptance

- Inclusion in PubMed, CAS, Scopus and Google Scholar

- Research which is freely available for redistribution

Submit your manuscript at www.biomedcentral.com/submit
C Biomed Central 